\title{
Redução da dor e ansiedade na vacinação: Revisão integrativa da literatura
}

\author{
Vaccination pain and anxiety reduction: Integrative literature review \\ Reducción del dolor y la ansiedade por vacunación: Revisión integradora de la literatura
}

Recebido: 25/05/2021 | Revisado: 01/06/2021 | Aceito: 23/06/2021 | Publicado: 09/07/2021

\author{
Érika Augusta Faria Maciel \\ ORCID: https://orcid.org/0000-0003-4648-0217 \\ Universidade de Itaúna, Brasil \\ E-mail: erikamaciel@uit.br \\ Beatriz Pereira Santos \\ ORCID: https://orcid.org/0000-0002-7749-3427 \\ Universidade de Itaúna, Brasil \\ E-mail: biap489@gmail.com \\ Emiliana Vasconcelos Oliveira Maciel \\ ORCID: https://orcid.org/0000-0001-7691-3867 \\ Universidade de Itaúna, Brasil \\ E-mail: millavmaciel@gmail.com \\ Simone Maria da Silva \\ ORCID: https://orcid.org/0000-0003-1345-5971 \\ Universidade de Itaúna, Brasil \\ E-mail: simonesilva.prado@yahoo.com.br \\ Thayane Vieira Carvalho \\ ORCID: https://orcid.org/0000-0001-8644-8954 \\ Clinica Auge, Brasil \\ E-mail: thayanevieira100@yahoo.com.br \\ Liliane Pena \\ ORCID: https://orcid.org/0000-0001-5521-1719 \\ Universidade de Itaúna, Brasil \\ E-mail: lilip85@yahoo.com.br \\ Regina Consolação dos Santos \\ ORCID: https://orcid.org/0000-0002-7393-3210 \\ Universidade de Itaúna, Brasil \\ E-mail: reginasantos72@outlook.com \\ Heber Paulino Pena \\ ORCID: https://orcid.org/0000-0002-9122-6827 \\ Universidade de Itaúna, Brasil \\ E-mail: heberppena@yahoo.com.br
}

\begin{abstract}
Resumo
A vacinação é imprescindível para a proteção imunológica dos indivíduos, principalmente nos seus primeiros anos de vida. Podemos afirmar que as vacinas são eficazes na prevenção de enfermidades, pela redução de doenças imunopreveníveis no mundo. O objetivo desse estudo é abordar as estratégias para redução da dor e do medo de vacinas em pacientes. Trata-se de revisão integrativa de literatura, incluindo coleta de dados através de levantamentos bibliográficos para a identificação das produções sobre o manuseio da dor durante a vacinação. Para o desenvolvimento desta revisão foram apresentadas de forma sucinta as 05 fases do processo de elaboração de uma revisão integrativa e as buscas realizadas na Biblioteca Virutal de Saúde (BVS) e PubMed. Uso de anestésicos locais, crioterapia, musicoterapia, uso de brinquedos terapêuticos, o aleitamento materno e participação dos pais foram algumas estratégias detectadas. A vacinação é uma das intervenções mais benéficas da saúde para salvar vidas. Cabe aos profissionais da área da saúde buscarem estratégias para garantir um atendimento de qualidade proporcionando assistência qualificada durante todo o processo da vacinação. O enfermeiro também deve estar atento aos sinais de ansiedade apresentados pelo paciente, assim como manter uma postura adequada para conduzir a situação. Portanto é necessário que o profissional tenha conhecimento e criatividade durante todo o processo de administração da vacina, assegurando o cuidado integral, com o foco único e exclusivo no paciente.
\end{abstract}

Palavras-chave: Dor; Imunização; Ansiedade; Medo; Agulhas.

\section{Abstract}

Vaccination is essential for the immunological protection of individuals, especially in their first years of life. We can say that vaccines are effective in preventing diseases, by reducing vaccine-preventable diseases in the world. The aim of this study is to address strategies for reducing pain and fear of vaccines in patients. This is an integrative literature review, including data collection through bibliographic surveys to identify productions on pain management during 
vaccination. For the development of this review, the 05 phases of the process of elaborating an integrative review and the searches carried out in the Virtual Health Library (VHL) and PubMed were presented. Use of local anesthetics, cryotherapy, music therapy, use of therapeutic toys, breastfeeding and parental participation were some of the strategies detected. Vaccination is one of the most beneficial health interventions to save lives. It is up to health professionals to seek strategies to ensure quality care, providing qualified care throughout the vaccination process. The nurse must also be aware of the signs of anxiety presented by the patient, as well as maintain an adequate posture to handle the situation. Therefore, it is necessary that the professional has knowledge and creativity throughout the vaccine administration process, ensuring comprehensive care, with a single and exclusive focus on the patient.

Keywords: Pain; Immunization; Anxiety; Fear; Needles.

\section{Resumen}

La vacunación es fundamental para la protección inmunológica de las personas, especialmente en sus primeros años de vida. Podemos decir que las vacunas son efectivas para prevenir enfermedades, al reducir las enfermedades prevenibles por vacunación en el mundo. El objetivo de este estudio es abordar estrategias para reducir el dolor y el miedo a las vacunas en los pacientes. Se trata de una revisión integradora de la literatura, que incluye la recopilación de datos a través de encuestas bibliográficas para identificar producciones sobre el manejo del dolor durante la vacunación. Para el desarrollo de esta revisión se presentaron las 05 fases del proceso de elaboración de una revisión integradora y las búsquedas realizadas en la Biblioteca Virtual en Salud (BVS) y PubMed. El uso de anestésicos locales, crioterapia, musicoterapia, uso de juguetes terapéuticos, lactancia materna y participación de los padres fueron algunas de las estrategias detectadas. La vacunación es una de las intervenciones sanitarias más beneficiosas para salvar vidas. Corresponde a los profesionales de la salud buscar estrategias que aseguren una atención de calidad, brindando una atención calificada durante todo el proceso de vacunación. El enfermero también debe estar atento a los signos de ansiedad que presenta el paciente, así como mantener una postura adecuada para manejar la situación. Por tanto, es necesario que el profesional tenga conocimiento y creatividad durante todo el proceso de administración de la vacuna, asegurando una atención integral, con un enfoque único y exclusivo en el paciente.

Palabras clave: Dolor; Inmunización; Ansiedad; Miedo; Agujas.

\section{Introdução}

A vacinação é imprescindível para a proteção imunológica dos indivíduos, principalmente nos seus primeiros anos de vida. Podemos afirmar que as vacinas são eficazes na prevenção de enfermidades, pela redução de doenças imunopreveníveis no mundo (Agarwal, 2011).

A vacinação é imprescindível para a proteção imunológica dos indivíduos, principalmente nos seus primeiros anos de vida. Podemos afirmar que as vacinas são eficazes na prevenção de enfermidades, pela redução de doenças imunopreveníveis no mundo (Brasil, 2020).

No Brasil tem se tornado referência na erradicação de doenças, com a proximidade da eliminação do sarampo e outros agravos que assolavam a população (Brasil, 2014). Para alcançar e manter esse benefício são necessárias estratégias para obter e manter alta cobertura vacinal, esse é o principal objetivo do Movimento Vacina Brasil, do Ministério da Saúde (Domingues, Fantinato; Duarte \& Garcia, 2019).

Dessa forma, é fundamental ampliar o acesso às informações baseadas em evidências, especialmente sobre os benefícios da vacinação. Além da divulgação nas mídias tradicionais e eletrônicas, tais estratégias devem contemplar a busca ativa de não vacinados nas populações-alvo, parcerias com escolas e universidades, ampliação dos horários de funcionamento dos postos de vacinação, mobilização da sociedade civil e colaboração das sociedades científicas em parceria com as três instâncias de gestão, bem como o estabelecimento de parcerias intra e intersetoriais (Domingues, Fantinato; Duarte \& Garcia, 2019).

Outras táticas salientadas são fomentar a produção de conhecimento, por meio de inquéritos de coberturas vacinais e de estudos acerca dos fatores associados à não vacinação, considerando-se as diferentes regiões do país, o que poderá contribuir na definição de estratégias complementares para o enfrentamento da situação atual (Domingues, Fantinato; Duarte \& Garcia, 2019). 
Neste contexto, salienta-se também a responsabilidade do profissional de saúde quanto ao armazenamento, preparação e administração de imunobiológicos, assim como o adequado acolhimento dos pacientes (Brasil, 2014).

Os profissionais de saúde devem estar preparados para lidar com indivíduos que apresentam ansiedade e desconforto ocasionado pelas vacinas injetáveis. Os procedimentos com agulhas geralmente causam medo, dor, pânico e fobia, principalmente se tratando de indivíduos altamente ansiosos (Domingues, Fantinato; Duarte \& Garcia, 2019).

Em alguns indivíduos a fobia pode persistir durante toda a vida, devido a um conjunto de comportamentos e características que influenciaram no desenvolvimento do medo patológico (Vasey \& Dadds, 2001; Weisz \& Kazdin, 2010).

Os profissionais da área da saúde devem ser previamente treinados sobre como monitorar, reconhecer e reduzir a ansiedade e a dor, a fim de acarretar adesão positiva, através de um tratamento humanizado. A abordagem adequada pode inclusive reverter experiências insatisfatórias anteriores (Vasey \& Dadds, 2001).

Há um crescente interesse relacionado a essa temática, o que torna relevante conhecer metodologias e técnicas de abordagem, assim como mecanismos de redução da dor. O objetivo desse estudo é abordar as estratégias para redução da dor e do medo de vacinas em pacientes.

\section{Metodologia}

Trata-se de revisão integrativa de literatura, incluindo coleta de dados através de levantamentos bibliográficos para a identificação das produções sobre o manuseio da dor durante a vacinação. Esse método tem a finalidade de dar um suporte para a tomada de decisões, permitindo melhor síntese de múltiplos estudos publicados, e possibilitando conclusões gerais a respeito de uma determinada área abordada (Mendes, Silveira \& Galvão, 2008, Souza, Silva \& Carvalho, 2010; Paranhos, Pina \& Mello, 2011).

Para o desenvolvimento desta revisão foram apresentadas de forma sucinta as 05 fases do processo de elaboração de uma revisão integrativa: 1) identificação do tema e seleção da questão de investigação de pesquisa; 2) estabelecimento de critérios de inclusão e exclusão de artigos (busca da literatura); 3) definição das informações a serem extraídas dos estudos selecionados; 4) avaliação dos estudos selecionados; 5) análise e síntese dos resultados e apresentação da revisão (Mendes, Silveira \& Galvão, 2008).

Foram realizadas buscas nas seguintes bases de dados: Biblioteca Virtual em Saúde (BVS) e Pubmed em agosto e setembro de 2020. Foram utilizados Descritores em Ciências da Saúde (DeCS), assim como o Medical Subject Headings (MeSH) e o operador booleano "AND". Para a busca dos artigos utilizamos os descritores em pares e trios e em suas combinações nas línguas portuguesa e inglesa: "Dor", "Imunização", "Ansiedade”, "Medo" e "Agulhas". Os critérios de inclusão foram artigos publicados na íntegra nos idiomas português, inglês e espanhol nos últimos dez anos. Foram excluídos os trabalhos que não se adequavam ao objetivo da pesquisa e estudos duplicados.

A partir dos critérios de inclusão e estratégias de busca foram identificados 253 trabalhos. Para seleção dos artigos, os títulos e resumos foram submetidos à leitura na íntegra e à análise minuciosa, foram excluídos 175 artigos por não abordarem a temática. Para elegibilidade, os 78 trabalhos restantes foram submetidos à leitura de texto completo, sendo excluídos 59 por não abordarem o assunto de investigação, restando 19 estudos, os quais foram selecionados. Os artigos foram obtidos na íntegra para categorização, avaliação e síntese dos estudos, conforme disposto no Diagrama de Seleção da Figura 1. 
Figura 1: Diagrama de seleção dos estudos.

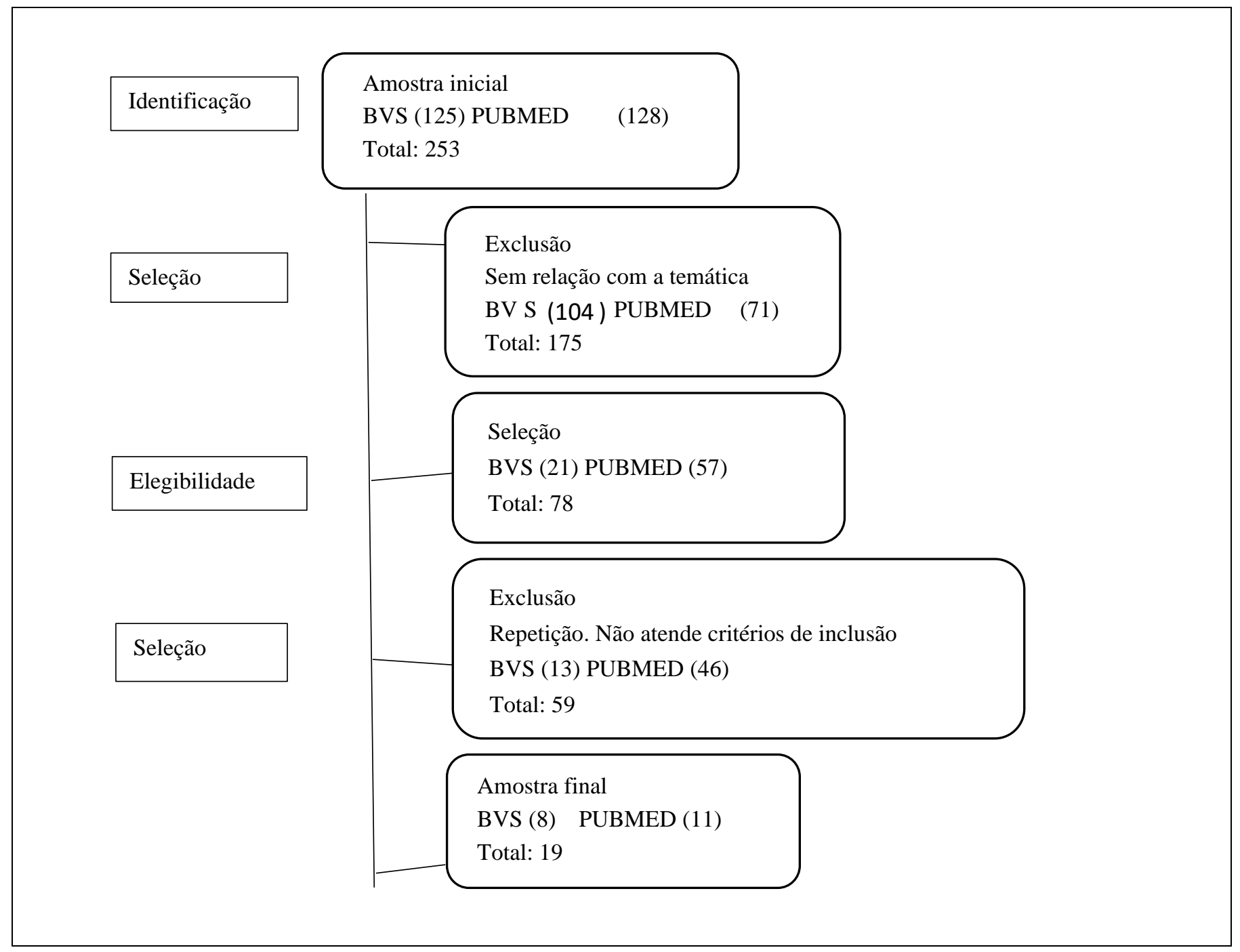

Fonte: Autores (2021).

\section{Resultados e Discussão}

A coleta e seleção dos artigos nas duas bases de dados resultaram na inclusão de 19 artigos para o presente estudo. Foram observados benefícios positivos nas práticas da utilização de métodos para amenizar a fobia, o medo e a ansiedade durante a aplicação do imunobiológico. Os resultados otimizaram a viabilidade da utilização de técnicas farmacológicas e não farmacológicas centrada no atendimento de forma humanizada, possibilitando um ganho considerável sobre a prática de vacinação e o conhecimento do problema.

Os artigos encontrados foram caracterizados em temas que abordaram de uma forma geral metodologias facilitadoras na redução da dor e ansiedade durante a vacinação. A dor pode ser definida como uma experiência humana não muito agradável, podendo gerar ativação do sistema nervoso somatossensorial após a ocorrência de uma lesão real ou potencial (Taddio, Chambers, Halperin, Ipp, Lockett, Riedes, et al., 2015).

O medo é uma reação normal e fisiológica do organismo desencadeada a partir de qualquer situação de ameaça ou perigo (Ollendick, 1983; Gullone, 2000). Quando identificado esse tipo de sentimento são necessários o apoio e a intervenção de profissionais e dos pais, quando vacinação em crianças, para que possam auxiliar na tentativa de reverter esse quadro. 


\section{Intervenções farmacológicas}

Os cremes anestésicos tópicos agem causando bloqueios reversíveis à condução ao longo das fibras nervosas que enviam sinais de dor e fornecem anestesia local, mas requerem um tempo mínimo de aplicação de 15 a 60 minutos antes da vacinação para atingir efeito ideal (Rogers \& Ostrow, 2004; Luthy, Beckstrand \& Pulsipher, 2013).

A utilização de spray anestésico à base de benzocaína associado a metodologias de distração, é um método de forma eficaz, rápida e barata de reduzir a dor e o desconforto durante o processo de vacinação em crianças de 4 a 6 anos (Stockwell, Irigoyen, Martinez \& Findley, 2011).

O EMLA é uma mistura eutética de anestésico local a 5\% (lidocaína 2,5\% e prilocaína 2,5\%) que é liberado nas camadas epidérmica e dérmica da pele e na proximidade dos receptores dérmicos da dor e terminações nervosas, que estabilizam as membranas neuronais inibindo os fluxos iónicos necessários para a iniciação e condução de impulsos, produzindo a anestesia local. É recomendado que seja administrado pelos pais antes da injeção, resultando significativamente a redução da dor após as injeções intramusculares (Taddio, Appleton, Bortolussi, Chambers, Dubey, Halperin, et al., 2010; Brenner, Rupp, Boucher, Weaver, Dusza \& Dokovoy, 2013; Taddio, Nulman, Goldbach, Ipp \& Koren, 1994).

\section{Intervenções não farmacológicas}

Medidas não farmacológicas para o alivio da dor em crianças estão sendo utilizadas nesses procedimentos para facilitar a continuidade do tratamento em razão da redução do estresse e da angustia nesses pacientes (Lander, Weltman \& So, 2006).

A crioterapia proporciona um tratamento à base do resfriamento do local que inicia a aplicação, reduzindo o estresse, angústia e a dor (IASP, 1994). Este mecanismo está relacionado ao compartilhamento de sinapses na medula espinhal, em que as fibras nervosas de condução do estímulo doloroso compartilham vias sinápticas com as fibras de condução térmicas, ativadas por termorreceptores (sensíveis à temperatura) e com as fibras de condução mecânicas, ativadas pelos mecanorreceptores (estimulados pela vibração). Essa interferência de resposta interneuronal promove a inibição da condução da informação dolorosa até a medula espinhal no sistema nervoso central, promovendo o alívio (Kakigi \& Shibasaki, 1992; Nahra \& Plaghki, 2005).

Outra metodologia de distração que vem ganhando espaço é a musicoterapia, que consiste em uma intervenção segura e inofensiva para reduzir a duração das crises de choro, melhorar a percepção da dor e os níveis de angústia durante os procedimentos de vacinação (Çetin \& Çevik, 2019; Yinger, 2016).

Com relação ao brinquedo terapêutico, este inclui ações de criatividade, lúdico e o humor, além de estimular a interação entre criança e o adulto. Do ponto de vista da criança, ele promove o desenvolvimento físico, psicológico, social e moral. Ajuda ainda, a revelar seus pensamentos e sentimentos, promovendo satisfação, diversão e espontaneidade, favorecendo o exercício de suas potencialidades. Além de ser uma forma de a criança "esquecer" a dor, o brinquedo pode revelar as "necessidades e sentimentos do pequeno paciente, auxiliando-o a compreender as situações ou procedimentos que irá submeter-se, estimulando-o a fique calmo e seguro (Taddio, Chambers, Halperin, Ipp, Lockett, Riedes, et al, 2015; Martins, Ribeiro, Borba \& Silva, 2001).

Já o Buzzy é um dispositivo reutilizável que combina frio externo e vibração, podendo ser utilizado tanto em adultos quando crianças. Sua principal função é a redução da dor durante a vacinação, diminuindo os altos níveis de ansiedade do paciente. O dispositivo é ligado e colocado sobre o local da aplicação durante 30 segundos antes da aplicação, em seguida é movido aproximadamente $5 \mathrm{~cm}$ proximal ao local, e mantido no lugar durante o restante do procedimento, diminuindo a ansiedade antes e durante a aplicação do imunobiológico (Schechter, Zempsky, Cohen, McGrath, McMurtry \& Bright, 2007; Czarnecki, Turner, Collins, Doellman, Wrona \& Reynolds, 2011). 
O leite materno é uma Intervenção natural, fácil e eficaz na diminuição da dor durante a vacinação, podendo ser facilmente adotadas por profissionais de saúde e pais (Shah, Aliwalas \& Shah, 2006). O leite contém agentes que têm propriedades analgésicas ou que pode ser convertido endogenamente em substâncias analgésicas, além de conter maior concentração de triptofano, precursor da melatonina, que serve para aumentar a concentração de beta endorfinas, que é um dos mecanismos para os efeitos nociceptivos do leite (Sparks, 2001; Agarwal, 2011).

\section{A influência dos pais na dor nos procedimentos}

Durante toda infância as crianças são submetidas a vários procedimentos para promover a saúde. Além disso, o comportamento dos pais pode resultar em aumento da ansiedade dos filhos durante todo processo de vacinação (Gidudu, Walco, Taddio, Zempsky, Halperin, Calugar, et al., 2012).

Os pais devem ser orientados sobre como administrar sua ansiedade durante experiências dolorosas dos filhos. Por exemplo, controlar a ansiedade utilizando intervenções como (respiração profunda e métodos que permitem o relaxamento), pode ajudar na diminuição do estresse parental e consecutivamente das crianças (Galvão, Pedroso \& Ramalho, 2015). Uma boa experiência durante a vacinação terá potencial para reduzir experiências negativas, diminuindo hesitações vacinais e, consequentemente, diminuindo a incidência de doenças evitáveis por vacinas (Mendes-Neto \& Santos, 2020; Taddio, McMurthy, Shah, Riddell, Chambers, Noel, et al., 2015).

\section{Considerações Finais}

A vacinação é uma das intervenções mais benéficas da saúde para salvar vidas. Devido a maioria das vacinas serem administradas pela via parenteral, esse procedimento pode ocasionar experiências negativas tanto físicas quanto psicológicas no paciente.

Existem diversas medidas que possibilitam reduzir a dor da aplicação de vacinas injetáveis e consequentemente a ansiedade. Foram identificadas diversas estratégias para redução da dor, como técnicas farmacológicas, com uso de anestésicos e não farmacológicas, utilizando crioterapia no local de aplicação da vacina, estimulação tátil, distração, entre outros. Salientase também a importância dos pais na redução de ansiedade dos filhos.

Cabe aos profissionais da área da saúde buscarem estratégias para garantir um atendimento de qualidade proporcionando assistência qualificada durante todo o processo da vacinação. O enfermeiro também deve estar atento aos sinais de ansiedade apresentados pelo paciente, assim como manter uma postura adequada para conduzir a situação. Portanto é necessário que o profissional tenha conhecimento e criatividade durante todo o processo de administração da vacina, assegurando o cuidado integral, com o foco único e exclusivo no paciente.

Espera-se que outros estudos sejam conduzidos no intuito de detectar novas estratégias assim como técnicas de comunicação terapêutica, inclusive para adultos.

\section{Referências}

Agarwal, R. (2011). Breastfeeding or breast milk for procedural pain in neonates: RHL commentary. The WHO Reproductive Health Library - World Health Organization.

Baxter, A. L., Cohen, L. L., McElvery, H. L., Lawson, M. L., \& von Baeyer, C. L. (2011). An integration of vibration and cold relieves venipuncture pain in a pediatric emergency department. Pediatr Emerg Care. 27(12), 1151-1156. 10.1097/PEC.0b013e318237ace4.

Brasil, Ministério da Saúde. (2020). Manual de vigilância epidemiológica de eventos adversos pós vacinação. Secretaria de Vigilância em Saúde, Departamento de Vigilância das Doenças Transmissíveis.

Brenner, S. M., Rupp, V., Boucher, J., Weaver, K., Dusza, S. W., \& Bokovoy, J. (2013). A randomized, controlled trial to evaluate topical anesthetic for 15 minutes before venipuncture in pediatrics. The American Journal of Emergency Medicine. 31(1), 20-25. 
Cobb, J. E., \& Cohen, L. L. (2009). A randomized controlled trial of the ShotBlocker for children's immunization distress. Clin Journal Pain. 25(9), 790-796. 10.1097/AJP.0b013e3181af1324.

Czarnecki, M. L., Turner, H. N., Collins, P. M., Doellman, D., Wrona, S., \& Reynolds, J. (2011). Procedural pain management: a position statement with clinical practice recommendations. Pain Management Nursing. 12(2), 95-111. 10.1016/j.pmn.2011.02.003.

Çetin, S. P., \& Çevik, K. Effects of vibration and cold application on pain and anxiety during intravenous catheterization. (2019). Journal of Perianesthesia Nursing. 34(4), 157-158.

Domingues, C. M. A. S., Fantinato, F. F. S. T., Duarte, E., \& Garcia, L. P. (2019). Vacina Brasil e estratégias de formação e desenvolvimento em imunizações. Epidemiologia de Serviços de Saúde. 28(2), 1-4.

Galvão, D. M. P. G., Pedroso, R. M. C. J., \& Ramalho, S. I. H. S. M. A. (2015). Intervenções não farmacológicas de redução da dor em uso na vacinação de lactentes non-pharmacological pain relief interventions used in infant vaccination. Revista INFAD - International Journal of Developmental and Educational Psychology. 1(1), 89-98. https://doi.org/10.17060/ijodaep.2015.n1.v1.254.

Gidudu, J. F., Walco, G. A., Taddio, A., Zempsky, W. T., Halperin, S. A., Calugar, A., et al. (2012). Immunization site pain: case definition and guidelines for collection, analysis, and presentation of immunization safety data. Vaccine. 4558-77.

Gullone, E. (2000). The development of normal fear: a century of research. Clin Psychol Rev. 20, 429-451.

IASP. Classification of Chronic Pain. 2nd ed. Merskey H, Bogduk N, editors. (1994). IASP Pain Terminology. 240p.

Kakigi, R., \& Shibasaki, H. (1992). Mechanisms of pain relief by vibration and movement. Journal Neurol Neurosurg Psychiatry. 55(1), 282-286.

Lander, J.A., Weltman, B. J., \& So, S. S. (2006). EMLA and amethocaine for reduction of children's pain associated with needle insertion. The Cochrane Database of Systematic Reviews. 1(3), 897-925. 10.1002/14651858.CD004236.pub2.

Luthy, K. E., Beckstrand, R. L., \& Pulsipher, A. (2013). Evaluation pf methods to relieve parental perceptions of vaccine-associated pain and anxiety in children: a pilot study. Journal of Pediatric Health Care. 27, 351-358.

Martins, M. R., Ribeiro, C. A., Borba, R. I. H., \& Silva, C. V. (2001). Protocol for the preparation of preschool children to venous puncture using therapeutic play. Revista Latino Americana de Enfermagem. 9(2), 76-85. https://doi.org/10.1590/S0104-11692001000200011.

Mendes, K. D. S., Silveira, R. C. C. P., \& Galvão, C. M. (2008). Revisão integrativa: método de pesquisa para a incorporação de evidências na saúde e na enfermagem. Texto Contexto Enfermagem. 17(4), 758-64.

Mendes-Neto, J. M., \& Santos, S. L. (2020). Vibração associada à crioterapia para alívio da dor em crianças. BrJP. 3(1), 53-57. https://doi.org/10.5935/25950118.20200012

Nahra, H., \& Plaghki, L. (2005). Innocuous skin cooling modulates perception and neurophysiological correlates of brief CO2 laser stimuli in humans. Eur Journal Pain. 9(1), 521-530.

Ollendick, T. H. (1983). Confiabilidade e validade do medo revisado cronograma de cirurgia para crianças (FSSC-R). Behav Res Ther. 21, 685-692.

Paranhos, V. D., Pina, J. C., \& Mello, D. F. (2011). Integrated management of childhood illness with the focus on caregives: an integrative literature review. Revista Latino Americano Enfermagem. 19(1), 203-211.

Rogers, T. L., \& Ostrow, C. L. (2004). The use of EMLA cream to decrease venipuncture pain in children. J Pediatr Nurs. 19, 33-39.

Schechter, N. L., Zempsky, W. T., Cohen, L. L., McGrath, P. J., McMurtry, C. M., Bright, N. S. (2007). Pain reduction during pediatric immunizations: Evidence based review and recommendations. Pediatrics. 119(5), 1184-98. 10.1542/peds.2006-1107.

Shah, P. S., Aliwalas, L. L., \& Shah, V. S. (2006). Breastfeeding or breast milk for procedural pain in neonates. Retrieved from Cochrane Database of Systematic Reviews. 10.1002/14651858.CD004950.pub2.

Souza, M. T., Silva, M. D., \& Carvalho, R. (2010). Revisão integrativa: o que é e como fazer. Einstein. 8(1), 102-106.

Sparks, L. (2001). Taking the "ouch" out of injections for children. Using distraction to decrease pain. MCN: The American Journal of Maternal/Child Nursing. 26(2), 72-78. 10.1097/00005721-200103000-00005

Stockwell, M., Irigoyen, M., Martinez, R., \& Findley, S. (2011). How parents' negative experiences at immunization visits affect child immunization status in a community in New York City. Public Health Reports. 24-32.

Taddio, A., Appleton, M., Bortolussi, R., Chambers, C., Dubey, V., Halperin, S., et al. (2010). Reducing the pain of childhood vaccination: na evidence-based clinical practice guideline. CMAJ-Best evidence, best practice, best health. 182, 43-55.

Taddio, A., Chambers, C. T., Halperin, S. A., Ipp, M., Lockett, D., Rieder, M. J., et al. (2015). Reducing pain during vaccine injections: clinical practice guideline. Clin ther. 31(2).

Taddio, A., McMurtry, C. M., Shah, V., Riddell, R. P., Chambers, C. T., Noel, M., et al. (2015). Reducing pain during vaccine injections: clinical practice guideline. CMAJ. 187(13), 975-982.

Taddio, A., Nulman, I., Goldbach, M., Ipp, M., \& Koren, G. (1994). Use of lidocaine-prilocaine cream for vaccination pain in infants. The Journal of Pediatrics. 124, 643-648.

Vasey, M. W., \& Dadds, M. R. (2001). A psicopatologia do desenvolvimento de ansiedade. Oxford University Press. 
Research, Society and Development, v. 10, n. 8, e15610816508, 2021

(CC BY 4.0) | ISSN 2525-3409 | DOI: http://dx.doi.org/10.33448/rsd-v10i8.16508

Weisz, J. R., \& Kazdin, A. E. (2010). Evidence-based psychotherapies for children and adolescents. Routledge.

Yinger, O. S. (2016). Music therapy as procedural support for young children undergoing immunizations: a randomized controlled study. Journal of music therapy. 31, 157-158. 